\title{
ALGEBRAIC CHARACTERIZATIONS IN COMPLEX DIFFERENTIAL GEOMETRY†
}

BY

\section{T. Y. THOMAS}

1. In the treatment of differential geometry from the modern invariantive standpoint it is usually unnecessary that the coordinates and the functions which define the structure of the space under consideration be real quantities. Adopting the more general hypothesis of complex coordinates and structure functions we arrive at the concept of generalized spaces of complex character. This procedure has the advantage that it serves to distinguish the purely formal aspects of the theory which are identical under the real or complex hypothesis from non-trivial questions of reality arising in the transition from the complex to the corresponding real space.

Let $S$ be a complex generalized space and denote by $F_{1}$ and $F_{2}$ systems of polynomials in the structure functions of $S$ and their derivatives to a certain order, the coefficients of these polynomials being definitely specified constants. We shall say that the conditions

$$
F_{1}=0, \quad F_{2} \neq 0
$$

constitute an algebraic characterization of a property $P$ of the space $S$ provided that necessary and sufficient conditions for the existence of the property $P$ are furnished by (1.1). Since the property $P$ is independent of the coordinate system adopted, the conditions (1.1) must be invariant under coordinate transformations.

In particular the equations $F_{1}=0$ alone may suffice for the algebraic characterization. This type of characterization may be described precisely as an algebraic characterization in terms of equations. We shall here be concerned exclusively with such characterizations, which for the sake of brevity will be referred to as simple algebraic characterizations. The results obtained will be seen to yield as immediate consequences certain algebraic characterizations which are not simple.

In the following discussion the above polynomials $F$ will be found directly as polynomials in the components of a complete set of tensor differential invariants of the space $S$. It will therefore be convenient to consider these components rather than the structure functions and their derivatives as the independent variables in the polynomials $F$.

† Presented to the Society, September 13, 1935; received by the editors November 20, 1934. 
Thus if $S$ is an affinely connected space, a projective space of paths, a metric space, or a conformal space, the vanishing of the corresponding curvature tensor gives a simple algebraic characterization of the flat space $S$. As is well known, the equations

$$
B_{\alpha \beta \gamma \delta}=K\left(g_{\alpha \delta} g_{\beta \gamma}-g_{\alpha \gamma} g_{\beta \delta}\right),
$$

in which the $B$ 's are the components of the curvature tensor and the $g$ 's the components of the fundamental metric tensor, express the conditions for a metric space to be of constant curvature $K$. The above equations do not constitute a strict algebraic characterization of the space of constant curvature since the constant $K$ is arbitrary. However, an algebraic characterization is obtained by elimination of $K$ which gives

$$
\left(g_{a d} g_{b c}-g_{a c} g_{b d}\right) B_{\alpha \beta \gamma \delta}-\left(g_{\alpha \delta} g_{\beta \gamma}-g_{\alpha \gamma} g_{\beta \delta}\right) B_{a b c d}=0 .
$$

Since the determinant $\left|g_{\alpha \beta}\right|$ does not vanish by hypothesis, not all of the expressions in parentheses in (1.3) will vanish as these are the second-order minors of $\left|g_{\alpha \beta}\right|$. Hence we can pass from (1.3) to (1.2) in which the quantity $K$ is at most a function of position. It then follows by Schur's theorem that $K$ is a constant and hence the equations (1.3) give an algebraic characterization of the metric spaces of constant curvature.

In a recent paper by $\mathrm{J}$. Levine and myself a proof of the existence of algebraic characterizations was given for a certain class of problems in differential geometry. $\dagger$ It was shown in particular that a simple algebraic characterization exists for the metric representations of an affinely connected space provided that the dimensionality of the representations is unspecified. We now give a more exhaustive treatment of this problem on the basis of the theory of algebraic manifolds and the Kronecker theory of algebraic elimination. Our methods are quite general and permit a wide range of application beyond the particular problem treated in this paper. We have shown that there exist $n$ irreducible algebraic manifolds which are of significance for our characterization problem. One of these irreducible manifolds furnishes a simple algebraic characterization of the 1-dimensional metric representations. The others give necessary conditions for the existence of representations of dimensionality $r>1$ but fail to meet the sufficiency requirement, with the result that none of these latter representations admit a simple algebraic characterization.

2. Consider the system of equations

$$
\frac{\partial g_{\alpha \beta}}{\partial x^{\gamma}}=g_{\sigma \beta} \Gamma_{\alpha \gamma}^{\sigma}+g_{\alpha \sigma} \Gamma_{\beta \gamma}^{\sigma}
$$

$\dagger$ T. Y. Thomas and J. Levine, On a class of existence theorems in differential geometry, Bulletin of the American Mathematical Society, vol. 40 (1934), p. 721. 
in the set of symmetric unknowns $g_{\alpha \beta}$ and the given components $\Gamma_{\beta \gamma}^{\alpha}(x)$ defining the (symmetric) connection of a complex space $S$ of $n(\geqq 2)$ dimensions. As integrability conditions of (2.1) we derive the following sequence:

$$
\begin{aligned}
g_{\sigma \alpha \alpha} B_{\beta \gamma \delta}^{\sigma}+g_{\beta \sigma} B_{\alpha \gamma \delta}^{\sigma} & =0, \\
g_{\sigma \alpha \alpha}^{\sigma} B_{\beta \gamma \delta, \epsilon}^{\sigma}+g_{\beta \sigma} B_{\alpha \gamma \delta, \epsilon}^{\sigma} & =0, \\
g_{\sigma \alpha} B_{\beta \gamma \delta, \epsilon, \zeta}^{\sigma}+g_{\beta \sigma} B_{\alpha \gamma \delta, \epsilon, \zeta}^{\sigma} & =0,
\end{aligned}
$$

where the $B_{\beta \gamma \delta}^{\alpha}, B_{\beta \gamma \delta, \epsilon}^{\alpha}, \cdots$ are the components of the curvature tensor and its successive covariant derivatives. It can be proved $\dagger$ that there exists an integer $N$ such that the vanishing of the resultant system $R(B)$ of the first $N$ sets of equations of the sequence (2.2) is necessary and sufficient for the existence of a solution of (2.1). If a solution $g_{\alpha \beta}(x)$ of (2.1) exists such that the rank of the matrix $\left\|g_{\alpha \beta}\right\|$ is $n$, then the $\Gamma_{\beta \gamma}^{\alpha}$ are Christoffel symbols with respect to the $g_{\alpha \beta}$ and the space $S$ is said to reduce to a metric space or to admit an $n$-dimensional metric representation. If the rank of the solution matrix $\left\|g_{\alpha \beta}\right\|$ is $r$, where $1 \leqq r \leqq n-1$, and if a metric is defined in the space $S$ by the degenerate quadratic differential form $g_{\alpha \beta} d x^{\alpha} d x^{\beta}$, the space will be multiply isomorphic to an $r$-dimensional metric space $S^{*}$, the metric of $S^{*}$ being defined by a form which is not degenerate; we then say that the space $S$ admits an $r$-dimensional metric representation. The equations $R(B)=0$ therefore give an algebraic characterization of the metric representations of the space $S$ under the hypothesis that the dimensionality of the representations is unspecified.

3. Now suppose that (2.1) admits a solution $g_{\alpha \beta}(x)$ for which the matrix $\left\|g_{\alpha \beta}\right\|$ is of rank $n$. We can then solve (2.1) for the $\Gamma_{\beta \gamma}^{\alpha}$ so as to express these quantities as Christoffel symbols

$$
\Gamma_{\beta \gamma}^{\alpha}=\frac{1}{2} g^{\alpha \sigma}\left(\frac{\partial g_{\sigma \beta}}{\partial x^{\gamma}}+\frac{\partial g_{\sigma \gamma}}{\partial x^{\beta}}-\frac{\partial g_{\beta \gamma}}{\partial x^{\sigma}}\right)
$$

in terms of the solution $g_{\alpha \beta}(x)$. Substituting (3.1) into the expression defining any component $B$ as a function of the $\Gamma$ 's and their derivatives it is seen that the component $B$ is given by an expression of the form

$$
B=\frac{P\left(g_{\alpha \beta}, \partial g_{\alpha \beta} / \partial x^{\gamma}, \cdots\right)}{\left|g_{\alpha \beta}\right|^{m}},
$$

where $P$ denotes a definitely determined polynomial, with rational coeffcients, in the $g_{\alpha \beta}$ and a finite number of their derivatives, and where $m$ is a

† See T. Y. Thomas and J. Levine, loc. cit., p. 721. 
suitable positive integer. If the $B$ 's occurring in the resultant system $R(B)$ have numerical values given by the parametric equations

$$
B=\frac{P\left(q_{\alpha \beta}, q_{\alpha \beta \gamma}, \cdots\right)}{\left|q_{\alpha \beta}\right|^{m}},
$$

where the $q$ 's are arbitrary subject to the symmetry conditions on the corresponding quantities in the right members of (3.2) and such that the determinant $\left|q_{\alpha \beta}\right|$ does not vanish, then $R(B)=0$, and the first $N$ sets of equations (2.2) admit a numerical solution; in fact $g_{\alpha \beta}=q_{\alpha \beta}$ is a solution of these equations. This follows from the fact that we can define a set of polynomial functions

$$
g_{\alpha \beta}(x)=q_{\alpha \beta}+q_{\alpha \beta \gamma} x^{\gamma}+\frac{1}{2} q_{\alpha \beta \gamma \delta} x^{\gamma} x^{\delta}+\cdots
$$

having the above quantities $q$ in (3.3) as coefficients and these functions can be used to determine the $\Gamma_{\beta_{\gamma}}^{\alpha}$ by means of (3.1). At $x^{\alpha}=0$ the resulting components $B$ will have values given by (3.3) and since (2.1) is merely another form of (3.1) for the case under consideration the first $N$ sets of equations (2.2), as integrability conditions of (2.1), will admit the above mentioned solution. Hence the number $N_{1}$ of algebraically independent components $B$ in $R(B)$ which correspond to a space $S$ admitting an $n$-dimensional metric representation is exactly determined by the parametric representation (3.3).

Let $\Delta$ denote a set of algebraically independent components $B$ appearing in the resultant system $R(B)$ without the restriction that the space $S$ admits an $n$-dimensional metric representation, the complete sets of identities of the $B$ 's in $S$ being used for the determination of these independent components. $\dagger$ If there are $N_{2}$ independent components $B$ in the set $\Delta$, we can interpret these as the coordinates of a space $E$ of $N_{2}$ dimensions. The inequality $N_{2}>N_{1}$ evidently holds.

Let $M_{n}$ denote the least algebraic manifold in $E$ defined by the parametric equations (3.3). By recourse to the theory of polynomial ideals it can be shown that $M_{n}$ is irreducible. $\neq$ The algebraic equations $F_{n}(B)=0$ which define $M_{n}$ are necessarily satisfied by the components $B$ of a space $S$ admitting an $n$-dimensional metric representation. Under such conditions we shall say as a matter of terminology that a space $S$ which admits an $n$-dimensional metric representation belongs to the manifold $M_{n}$. Since $M_{n}$ is the least algebraic manifold satisfying the required conditions given by (3.3) the equations $F_{n}(B)=0$ must give a simple algebraic characterization of the $n$-dimensional metric representations of $S$ if such a characterization exists.

$\dagger$ See T. Y. Thomas, The Differential Invariants of Generalized Spaces, Cambridge University Press, 1934, p. 134.

$\ddagger$ B. L. van der Waerden, Moderne Algebra, II, Berlin, Springer, 1931, p. 58. 
4. We shall now extend the above discussion to the case of the $r$-dimensional metric representations of $S$ where it is assumed that $r<n$. For this purpose we suppose that (2.1) admits a solution $g_{\alpha \beta}(x)$ with matrix $\left\|g_{\alpha \beta}\right\|$ of rank $r$. It is then possible to make a non-singular coordinate transformation $x \rightarrow y$ such that $\left\|g_{\alpha \beta}\right\|$ assumes the form

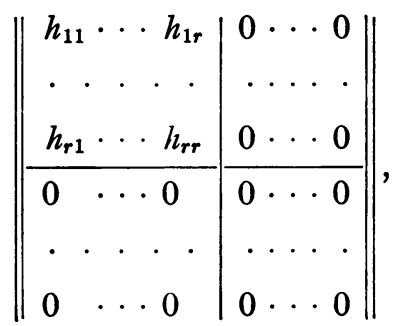

where the quantities $h_{\alpha \beta}$ depend on the variables $y^{1}, \cdots, y^{r}$ alone. If $C_{\beta \gamma}^{\alpha}$ denote the components of the connection with respect to the $y$ coordinate system, then these components must vanish for $\alpha=1, \cdots, r ; \beta=r+1, \cdots, n$; $\gamma=1, \cdots, n$. The components $C_{\beta \gamma}^{\alpha}$ for $\alpha>r$ and $\beta, \gamma=1, \cdots, n$ are arbitrary analytic functions of the coordinates $y^{1}, \cdots, y^{n}$. The remaining components $C_{\beta \gamma}^{\alpha}$ where $\alpha, \beta, \gamma=1, \cdots, r$ are Christoffel symbols with respect to the $h$ 's in the $r$ th-order minor in the upper left hand corner of the above matrix, $\dagger$ i.e.,

$$
\left.C_{\beta \gamma}^{\alpha}=\frac{1}{2} h^{\alpha \sigma}\left(\frac{\partial h_{\sigma \beta}}{\partial y^{\gamma}}+\frac{\partial h_{\sigma \gamma}}{\partial y^{\beta}}-\frac{\partial h_{\beta \gamma}}{\partial y^{\sigma}}\right) \quad \text { (indices }=1, \cdots, r\right) .
$$

If we denote by $D$ the components $B$ when taken with respect to the $y$ coordinates, we obtain a parametric representation of the $D$ 's corresponding to (3.3) on the basis of the conditions (4.1) and the fact that certain of the $C$ 's vanish while others are completely arbitrary; in this representation the nonvanishing determinant $\left|q_{\alpha \beta}\right|$ of the $r$ th order will occur in place of the corresponding $n$ th-order determinant in the denominators of (3.3). To obtain the parametric representation with respect to the arbitrary $x$ coordinate system we have merely to transform the above expressions for the $D$ 's into the $B$ 's by the tensor transformations, i.e.,

$$
B_{\beta \gamma \delta}^{\alpha}=D_{\nu \sigma \tau}^{\mu} u_{\beta}^{\gamma} u_{\gamma}^{\sigma} u_{\delta}^{\tau} \nu_{\mu}^{\alpha}, \cdots,
$$

$\dagger$ In particular if $r=1$, these equations assume the form

$$
C_{11}^{1}=\frac{1}{2} \cdot \frac{1}{h_{11}} \cdot \frac{\partial h_{11}}{\partial y^{1}} .
$$

See T. Y. Thomas and J. Levine, loc. cit., p. 721. 
where the $u_{\beta}^{\alpha}$ are arbitrary parameters such that the determinant $\left|u_{\beta}^{\alpha}\right|$ is not equal to zero and the $v_{\beta}^{\alpha}$ are their normalized cofactors.

If we denote by $M_{r}$ the least algebraic manifold in $E$ defined by (4.2) this manifold is irreducible as was the case for the manifold $M_{n}$, and the algebraic equations $F_{r}(B)=0$ which define $M_{r}$ constitute necessary conditions on the space $S$ for this space to admit an $r$-dimensional metric representation. If a simple algebraic characterization exists it must evidently be given by these equations.

TheоRем. There exist $n$ irreducible algebraic manifolds $M_{1}, \cdots, M_{n}$ such that any space $S$ which admits an r-dimensional metric representation belongs to the manifold $M_{r}$.

5. We shall now prove a lemma which will have application in the follow ing section.

LEMMA. If $r$ is any integer of the set $1, \cdots, n-1$ and if the solution matrix $\left\|g_{\alpha \beta}\right\|$ of (2.1) is of rank $r$ at a point $x^{\alpha}=p^{\alpha}$ of its domain of definition, then there exists a neighborhood of $p^{\alpha}$ in which $\left\|g_{\alpha \beta}\right\|$ is of rank $r$.

Take first the case $r=n-1$. For the derivative of the determinant $\left|g_{\alpha \beta}\right|$ of the matrix $\left\|g_{\alpha \beta}\right\|$ we have

$$
\frac{\partial\left|g_{\mu \nu}\right|}{\partial x^{\gamma}}=\frac{\partial g_{\alpha \beta}}{\partial x^{\gamma}} A^{\alpha \beta},
$$

where $A^{\alpha \beta}$ denotes the cofactor of the corresponding element $g_{\alpha \beta}$ of the matrix $\left\|g_{\alpha \beta}\right\|$. Substituting from (2.1), equations (5.1) become

$$
\frac{\partial\left|g_{\mu \nu}\right|}{\partial x^{\gamma}}=\left(g_{\sigma \beta} \Gamma_{\alpha \gamma}^{\sigma}+g_{\alpha \sigma} \Gamma_{\beta \gamma}^{\sigma}\right) A^{\alpha \beta}=2\left|g_{\mu \nu}\right| \Gamma_{\sigma \gamma}^{\sigma} .
$$

Since $\left|g_{\mu \nu}\right|=0$ at $x^{\alpha}=p^{\alpha}$ the left member of (5.2) vanishes at $x^{\alpha}=p^{\alpha}$ and consequently successive derivatives of the determinant $\left|g_{\mu \nu}\right|$ will likewise vanish at $x^{\alpha}=p^{\alpha}$. By hypothesis the rank of $\left\|g_{\alpha \beta}\right\|$ is $r=n-1$ at $x^{\alpha}=p^{\alpha}$ and hence the rank of this matrix is $n-1$ in a certain neighborhood of $p^{\alpha}$.

If $r<n-1$ we consider the determinant $\left|g_{\mu \nu}\right|$ in the left member of (5.1) to be any determinant of order $r+1$ selected from the matrix $\left\|g_{\alpha \beta}\right\|$, the $A^{\alpha \beta}$ being the cofactors of corresponding elements of the determinant $\left|g_{\mu \nu}\right|$. Then (5.1) is satisfied, and making the substitution (2.1) we obtain the first set of equations (5.2), in which the indices $\alpha, \beta$ are now restricted by the particular selection of the $(r+1)$ st-order determinant $\left|g_{\mu \nu}\right|$. The right members of these latter equations now expand into a linear and homogeneous expression in determinants of order $r+1$ of the matrix $\left\|g_{\alpha \beta}\right\|$. Hence the rank of $\left\|g_{\alpha \beta}\right\|$ is $r$ in a neighborhood of $x^{\alpha}=p^{\alpha}$ and the above lemma is proved. 
6. Suppose that $S$ belongs to $M_{r}$. We shall then show that $S$ admits a metric representation of dimensionality $m \leqq r$. To prove this result we make use of the theorem on resultant systems of homogeneous algebraic equations. $\dagger$

Denote by $L_{n-1}(B)=0$ the resultant system of the first $N$ sets of equations (2.2) combined with the determinant equation

$$
\left|\begin{array}{c}
g_{11} \cdots \cdot g_{1 n} \\
\cdot \cdots \cdot \cdot \\
g_{n 1} \cdots \cdot g_{n n}
\end{array}\right|=0 .
$$

More generally denote by $L_{r}(B)=0$ for $r=1, \cdots, n-1$ the resultant system of the first $N$ sets of equations (2.2) and the set of equations obtained by equating to zero all determinants of order $r+1$ in the left member of (6.1). Then $L_{r}(B)=0$ will define an algebraic manifold $G_{r}$ in the space $E$. The manifold defined in $E$ by the resultant system $R(B)=0$ of the first $N$ sets of equations (2.2) will be denoted by $G_{n}$. It is evident from the equations of definition of the manifolds $G_{r}$ for $r=1, \cdots, n$ that

$$
G_{n} \supset G_{n-1} \supset \ldots \supset G_{1} \text {. }
$$

Now $M_{n} \subset G_{n}$. Since the coordinates $B$ of any point $P$ of $M_{n-1}$ having a parametric representation of the form (4.2) permit a solution of the first $N$ sets of equations (2.2) such that (6.1) is satisfied it follows that $P$ lies in $G_{n-1}$. If the point $P$ of $M_{n-1}$ does not have the parametric representation (4.2) used in the determination of $M_{n-1}$ it follows from a theorem in the theory of algebraic manifolds that $P$ is the limit of a sequence of points $P_{1}, P_{2}, \cdots$ of $M_{n-1}$ having this representation $\ddagger$ and hence must likewise belong to $G_{n-1}$, i.e., $M_{n-1} \subset G_{n-1}$. Continuing we obtain the set of relations

$$
M_{n} \subset G_{n}, M_{n-1} \subset G_{n-1}, \cdots, M_{1} \subset G_{1} \text {. }
$$

Suppose that $S$ belongs to the manifold $G_{r}$ where $r$ is an integer of the set $1, \cdots, n$. The equations $(2.1)$ will therefore admit a solution and hence there exists an integer $N^{*}$ such that the first $N^{*}$ sets of equations (2.2), which we shall call the equations $H$ for brevity, are algebraically consistent and all their solutions satisfy the $\left(N^{*}+1\right)$ st set of these equations. Let $x^{\alpha}=p^{\alpha}$ denote a

$\dagger$ B. L. van der Waerden, loc. cit., p. 14.

‡ See J. F. Ritt, Differential Equations from the Algebraic Standpoint, Colloquium Publications of the American Mathematical Society, vol. 14, New York, 1932, p. 91. Also B. L. van der Waerden, Zur algebraischen Geometrie, III, Mathematische Annalen, vol. 108 (1933), p. 694. 
point of $S$ where the rank of the matrix of $H$ has its greatest value. Denote by $(B)_{p}$ the values of the corresponding $B$ 's in $H$. These equations, in which the coefficients $B$ have the values $(B)_{p}$, will possess a solution $g_{\alpha \beta}=\left(g_{\alpha \beta}\right)_{p}$ such that the rank of the matrix $\left\|\left(g_{\alpha \beta}\right)_{p}\right\|$ is $m \leqq r$ since the resultant system $L_{r}(B)=0$ is satisfied by the values $(B)_{p}$. Since the rank of the matrix of the equations $H$ for $B$ 's in a certain neighborhood $\Delta^{*}$ of $x^{\alpha}=p^{\alpha}$ is the same as when the $B^{\prime}$ 's have the values $(B)_{p}$, it follows that the general solution $g_{\alpha \beta}$, valid in $\Delta^{*}$, will yield the values $\left(g_{\alpha \beta}\right)_{p}$ for $x^{\alpha}=p^{\alpha}$ and for the selection $\left(g_{\alpha \beta}\right)_{p}$ of the values of those $g_{\alpha \beta}$ which enter as arbitrary quantities in the general solution. Now in accordance with the theory of equations of the type $(2.1), \dagger$ these arbitrary quantities $g_{\alpha \beta}$ are to be determined as the solutions of a completely integrable system of differential equations, the general solution of (2.1) then being obtainable as the algebraic solution of $H$. Hence there exists a solution $g_{\alpha \beta}$ of (2.1) such that $g_{\alpha \beta}=\left(g_{\alpha \beta}\right)_{p}$ at $x^{\alpha}=p^{\alpha}$. By the lemma of $\S 5$ the matrix $\left\|g_{\alpha \beta}\right\|$ of this solution will be of rank $m \leqq r$ in the neighborhood $\Delta^{*}$ of $x^{\alpha}=p^{\alpha}$. Hence if $S$ belongs to $G_{r}$ the space $S$ will admit a metric representation of dimensionality $m \leqq r$. Since $M_{r} \subset G_{r}$ by (6.3) we have the following result:

THEOREM. If the space $S$ belongs to the irreducible manifold $M_{r}$ when $r$ is any integer of the set $1, \cdots, n$, it will admit a metric representation of dimensionality $m \leqq r$.

This theorem when combined with the theorem of $\$ 4$ gives the following

Corollary. A necessary and sufficient condition for the space $S$ to admit a 1-dimensional metric representation is that it belong to the irreducible manifold $M_{1}$.

It is evident from the above that the manifold $M_{1}$ can be replaced by the manifold $G_{1}$ in the statement of this corollary. Hence the resultant system $L_{1}(B)=0$ gives an algebraic characterization of the 1-dimensional metric representations of $S$.

7. It will now be shown that the equations $F_{r}(B)=0$ which define the manifold $M_{r}$ where $r$ has any value of the set 2, . , n are not sufficient to insure the existence of an $r$-dimensional metric representation of $S$, and hence in accordance with the observation of $\$ 4$ that the $r$-dimensional metric representations of $S$ do not admit a simple algebraic characterization.

Consider a one-parameter family of $n$-dimensional metric spaces $S_{a}$ the components of the fundamental metric tensors of which are given by the matrix

† See, for example, T. Y. Thomas, Differential Invariants of Generalized Spaces, p. 202. 


$$
\left\|\begin{array}{ccccc}
\sin a \phi & 0 & \cdots & \cdot & 0 \\
0 & e^{a \phi} & \cdots & \cdot & 0 \\
\cdot & \cdot & \cdots & \cdot & \cdot \\
0 & 0 & \cdots & 0 & e^{a \phi}
\end{array}\right\|,
$$

where $a$ denotes the (real) parameter and $\phi$ is an analytic function of the coordinates $x^{1}, \cdots, x^{n}$ in the neighborhood of the values $x^{\alpha}=0$ at which the function $\phi$ is assumed to be different from zero; all other components of the tensors except those appearing in the diagonal of the matrix vanish identically. The spaces $S_{a}$ are thus defined for values of the parameter $a$ different from zero. Calculation of the resulting Christoffel symbols give

$$
\begin{array}{ll}
\left\{\begin{array}{c}
J \\
I I
\end{array}\right\}=-\frac{a}{2} \frac{\partial \phi}{\partial x^{J}}, & \left\{\begin{array}{c}
J \\
11
\end{array}\right\}=-\frac{a}{2}\left(\frac{\cos a \phi}{e^{a \phi}}\right) \frac{\partial \phi}{\partial x^{J}}, \\
\left\{\begin{array}{c}
1 \\
I I
\end{array}\right\}=-\frac{a}{2}\left(\frac{e^{a \phi}}{\sin a \phi}\right) \frac{\partial \phi}{\partial x^{1}}, & \left\{\begin{array}{c}
1 \\
1 J
\end{array}\right\}=\frac{a}{2}\left(\frac{a}{\tan a \phi}\right) \frac{\partial \phi}{\partial x^{J}}, \\
\left\{\begin{array}{c}
I \\
1 I
\end{array}\right\}=\frac{a}{2} \frac{\partial \phi}{\partial x^{1}}, & \left\{\begin{array}{c}
I \\
I J
\end{array}\right\}=\frac{a}{2} \frac{\partial \phi}{\partial x^{J}}, \\
\left\{\begin{array}{c}
I \\
I I
\end{array}\right\}=\frac{a}{2} \frac{\partial \phi}{\partial x^{I}}, & \left\{\begin{array}{c}
1 \\
11
\end{array}\right\}=\frac{1}{2}\left(\frac{a}{\tan a \phi}\right) \frac{\partial \phi}{\partial x^{1}},
\end{array}
$$

where $I, J=2, \cdots, n$ and the remaining symbols vanish identically. Now let $a \rightarrow 0$ and denote the limiting values of the Christoffel symbols $\left\{\begin{array}{c}\boldsymbol{\alpha} \gamma \\ \boldsymbol{\beta} \gamma\end{array}\right\}$ by the corresponding symbols $\Gamma_{\beta \gamma}^{\alpha}$. Then those symbols in (7.2) which do not approach zero become

$$
\left.\begin{array}{l}
\Gamma_{I I}^{1}=-\frac{1}{2} \theta_{1} \\
\Gamma_{1 \alpha}^{1}=\frac{1}{2} \theta_{\alpha}
\end{array}\right\} \text { where } \theta=\log \phi, \theta_{\alpha}=\frac{\partial \theta}{\partial x^{\alpha}} .
$$

Denote by $S^{*}$ the affinely connected space for which the components of connection $\Gamma_{I I}^{1}$ and $\Gamma_{1 \alpha}^{1}$ are given by (7.3), the remaining components being zero identically.

Now observe that the Christoffel symbols for the spaces $S_{a}$ can be represented by expansions of the form

$$
\psi_{0}+\psi_{1} a+\psi_{2} a^{2}+\cdots,
$$

where the $\psi$ 's are analytic functions of the coordinates $x^{\alpha}$ in the neighborhood of $x^{\alpha}=0$. The functions represented by $\psi_{0}$ in these expansions are the components of the connection of the space $S^{*}$. Hence the components $B$ of the curvature tensor and its successive covariant derivatives for the spaces $S_{a}$ 
approach, as $a \rightarrow 0$, the corresponding components $B$ for the space $S^{*}$. Since the spaces $S_{a}$ are such that the equations $F_{n}(B)=0$ which define the manifold $M_{n}$ are satisfied, it follows that these equations are likewise satisfied for the space $S^{*}$, i.e. the space $S^{*}$ belongs to the algebraic manifold $M_{n}$.

Now consider the equations (2.1) in which the $\Gamma$ 's are the components of connection of the space $S^{*}$. The first set of integrability conditions (2.2) then reduces to

$$
g_{\alpha 1} B_{\beta \gamma \delta}^{1}+g_{\beta 1} B_{\alpha \gamma \delta}^{1}=0 .
$$

Take $\alpha=\beta=\gamma=I$ where $I=2, \cdots, n$ and $\delta=1$. Then (7.4) becomes

$$
g_{I 1} B_{I I 1}^{1}=0, \text { or } g_{I 1}\left(\frac{\partial \theta_{I}}{\partial x^{I}}+\frac{\partial \theta_{1}}{\partial x^{1}}+\frac{1}{2} \theta_{I}^{2}+\frac{1}{2} \theta_{1}^{2}\right)=0,
$$

where it is of course understood that no summation is involved in these equations. Hence if we choose the function $\phi$ so that

$$
\frac{\partial \theta_{I}}{\partial x^{I}}+\frac{\partial \theta_{1}}{\partial x^{1}}+\frac{1}{2} \theta_{I}^{2}+\frac{1}{2} \theta_{1}^{2} \neq 0 \quad(I=2, \cdots, n),
$$

it follows that $g_{I 1}=0$. Now take $\alpha=\delta=1$, and $\beta=\gamma=I$ in (7.4); then these equations become

$$
g_{11}\left(\frac{\partial \theta_{I}}{\partial x^{I}}+\frac{\partial \theta_{1}}{\partial x^{1}}+\frac{1}{2} \theta_{I}^{2}+\frac{1}{2} \theta_{1}^{2}\right)=0,
$$

and hence the above condition (7.5) likewise gives $g_{11}=0$. It now follows from the equations (2.1) that $\partial g_{I J} / \partial x^{\alpha}=0$ for $\alpha=1, \cdots, n$ and $I, J=2, \cdots, n$, so that the most general possible solution of these equations under the condition (7.5) is that given by the matrix

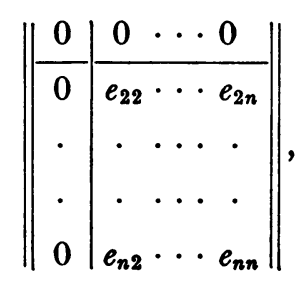

where the $e$ 's are arbitrary constants. Conversely it is seen immediately that this matrix represents a solution of (2.1) for the case under consideration. Hence the space $S^{*}$ does not admit an $n$-dimensional metric representation although it belongs to the manifold $M_{n}$. It follows that the n-dimensional 
metric representations of an affinely connected space $S$ do not admit a simple algebraic characterization. $\dagger$

8. To extend this result to the case of the $r$-dimensional metric representations for $r=2, \cdots, n-1$ we can consider a set of symmetric quantities $h_{\alpha \beta}$, where $\alpha, \beta=1, \cdots, r$, defined by a matrix of the form (7.1) in which the function $\phi$ depends on the coordinates $y^{1}, \cdots, y^{r}$ alone. We then use the equations (4.1) to define the components $C_{\beta \gamma}^{\alpha}$ for $\alpha, \beta, \gamma=1, \cdots, r$ and take the remaining $C$ 's subject to the restrictions stated in $\$ 4$. Allowing the parameter $a$ to approach zero we obtain a set of functions $\Gamma$ defining the connection of a space $S^{* *}$ which must belong to the manifold $M_{r}$, although a consideration of the equations (2.1) for $S^{* *}$ shows that this space, subject to restrictions corresponding to (7.4), can admit at most an $(r-1)$-dimensional metric representation. While this process is thus analogous to that carried out in $\$ 7$ it is desirable nevertheless to give the details of the process since certain formal difficulties present themselves. $\ddagger$

For definiteness in our discussion we shall employ only the following letters as indices with the ranges indicated:

$$
\begin{aligned}
I, J, K & =2, \cdots, r, \\
h, i, j, k, l & =r+1, \cdots, n, \\
\alpha, \beta, \gamma, \sigma & =1, \cdots, r, \\
\Lambda, \Theta, \Phi, \Psi, \Omega & =1, \cdots, n .
\end{aligned}
$$

Corresponding to (7.2) the limiting values are now given by

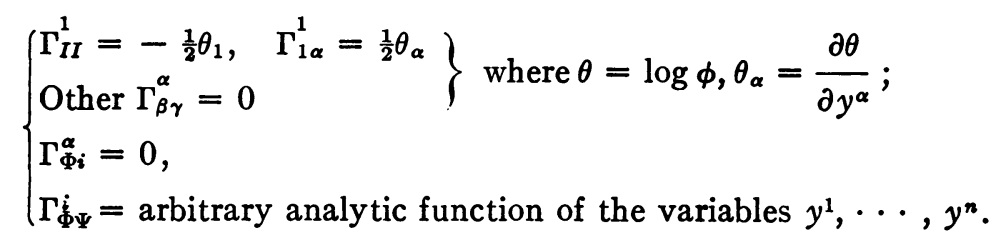

Using these values of the $\Gamma$ 's we find that

$$
\begin{aligned}
& B_{1 \alpha I}^{1}=B_{\alpha \beta \gamma}^{I}=B_{\alpha \beta i}^{I}=B_{i \beta \gamma}^{\alpha}=B_{i \beta j}^{\alpha}=B_{\Phi j k}^{\alpha}=0, \\
& B_{j k l}^{i}=\frac{\partial \Gamma_{j k}^{i}}{\partial y^{l}}-\frac{\partial \Gamma_{j l}^{i}}{\partial y^{k}}+\Gamma_{h l}^{i} \Gamma_{j k}^{h}-\Gamma_{h k}^{i} \Gamma_{j l}^{h} .
\end{aligned}
$$

$\dagger$ The method employed to deduce this result shows that necessary and sufficient conditions for the existence of an $n$-dimensional metric representation of the space $S$ can not be expressed by any system of equations of the form $F(B)=0$ the left members of which are continuous functions of the components $B$. As the algebraic characterization is however of primary interest we have limited the above statement to such characterizations.

$\ddagger$ I am indebted to Dr. J. Levine for the details of the treatment given in this section. 
Now consider the first set of integrability conditions (2.2) of the equations (2.1) determined by (8.1), namely

$$
g_{\Phi \Omega} B_{\Psi \Delta \Theta}^{\mathbb{Q}}+g_{\Omega \Psi} B_{\Psi \Delta \Theta}^{\mathbb{Q}}=0 .
$$

Taking $\Phi, \Psi, \Lambda, \Theta=i, j, k, l$ respectively and making use of (8.2), these equations become

$$
g_{i h} B_{j k l}^{h}+g_{j h} B_{i k l}^{h}=0 .
$$

In these latter equations put $i=j$ so as to obtain

$$
g_{i h} B_{i k l}^{h}=0
$$

( $i$ not summed).

Put $W=n-r$. Then in (8.6) for each value of $i$ we have $W(W-1) / 2$ equations in the $W$ unknowns $g_{i h}$.

Case I. $W \geqq 3$. Since $W \leqq W(W-1) / 2$ we can form from the matrix of the coefficients $g_{i h}$ in (8.6) the following determinant of order $W$ :

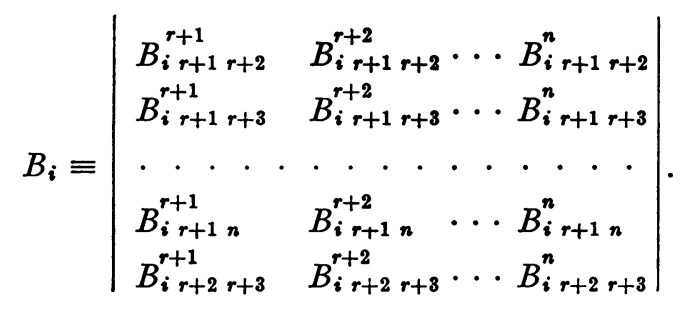

None of the determinants $B_{i}$ is identically zero since the elements $B$ in any determinant $B_{i}$ are algebraically independent quantities; this follows by recourse to the complete set of identities satisfied by the $B$ 's as defined by (8.3) in terms of the arbitrary functions $\Gamma_{j k}^{i}$ in (8.1). Hence we can choose the $\Gamma_{j k}^{i}$ so that $B_{i} \neq 0$ for $i=r+1, \cdots, n$. Hence $g_{i h}=0$ in consequence of (8.6).

From (8.4) we now obtain

$$
g_{\alpha h} B_{i k l}^{h}=0,
$$

use being made of the fact that the above quantities $g_{i n}$ are equal to zero. Hence $g_{\alpha h}=0$ since a determinant $B_{i}$ is contained in the matrix of the coefficients of (8.7).

Case II. $W=2$. From (8.4) select the following three equations:

$$
\begin{aligned}
& g_{m m} B_{m m n}^{m}+g_{m n} B_{m m n}^{n}=0, \\
& g_{n m} B_{n m n}^{m}+g_{n n} B_{n m n}^{n}=0, \\
& g_{n n} B_{m m n}^{n}+g_{m n}\left(B_{m m n}^{m}+B_{n m n}^{n}\right)+g_{m m} B_{n m n}^{m}=0,
\end{aligned}
$$


where $m$ is used to denote $n-1$. Hence if the arbitrary $\Gamma$ 's in (8.1) are chosen so that

$$
\begin{aligned}
& \left|\begin{array}{lll}
B_{m m n}^{m} & B_{m m n}^{n} & 0 \\
0 & B_{n m n}^{m} & B_{n m n}^{n} \\
B_{n m n}^{m} & B_{m m n}^{m}+B_{n m n}^{n} & B_{m m n}^{n}
\end{array}\right| \\
& \quad=\left(B_{m m n}^{m}+B_{n m n}^{n}\right)\left(B_{n m n}^{m} B_{m m n}^{n}-B_{m m n}^{m} B_{n m n}^{n}\right) \neq 0,
\end{aligned}
$$

it will follow that $g_{m m}=g_{m n}=g_{n n}=0$. Now from (8.7) we obtain

$$
\begin{gathered}
g_{\alpha m} B_{m m n}^{m}+g_{\alpha n} B_{m m n}^{n}=0, \\
g_{\alpha m} B_{n m n}^{m}+g_{\alpha n} B_{n m n}^{n}=0,
\end{gathered}
$$

the determinant of which is a factor of the above determinant (8.8). Hence $g_{\alpha m}=g_{\alpha n}=0$.

Case III. $W=1$. From (8.4) we obtain

$$
g_{n n} B_{n \alpha \beta}^{n}=0 .
$$

Choosing the arbitrary $\Gamma$ 's in (8.1) so that not all the coefficients $B_{n a \beta}^{n}$ are equal to zero, we have $g_{n n}=0$. Hence from (8.4) we have

$$
g_{\alpha n} B_{n \beta n}^{n}=0,
$$

from which $g_{\alpha n}=0$ can be obtained.

We have now shown that the arbitrary $\Gamma$ 's in (8.1) can be chosen so that the solution matrix of (2.1) will have the form

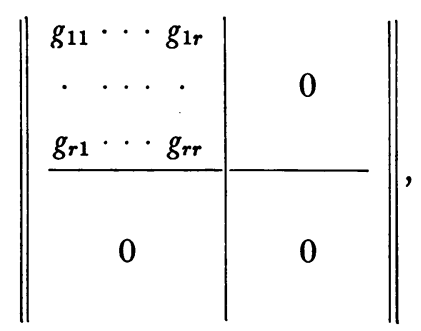

where $r$ has any value in the set $2, \cdots, n-1$. To make the rank of this matrix be $<r$ we now make $g_{1 \alpha}=0$. By a suitable selection of the indices in (8.4) these equations give

$$
g_{11}\left(\frac{\partial \theta_{I}}{\partial y^{J}}+\frac{1}{2} \theta_{I} \theta_{J}\right)=0, \quad g_{k 1}\left(\frac{\partial \theta_{1}}{\partial y^{J}}+\frac{1}{2} \theta_{1} \theta_{J}\right)=0 .
$$


Hence to have $g_{1 \alpha}=0$ we have merely to choose $\phi$ so that one of the coefficients in each of these two sets of equations is different from zero. As in $\$ 7$ the most general solution of the equations (2.1) is thus seen to be represented by the matrix

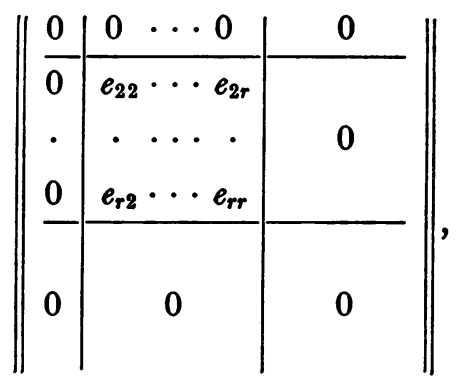

where the $e$ 's are arbitrary constants. This gives the following theorem which includes the result of $\$ 7$.

THEOREM. The r-dimensional metric representations of an afinely connected space $S$, where $r$ is an integer of the set $2, \cdots, n$, do not admit a simple algebraic characterization.

Princeton UntVersity,

Princeton, N. J. 